\title{
Effect of a Compatibilizer on the Microstructure and Properties of Partially Biodegradable LDPE/Aliphatic Polyester/Organoclay Nanocomposites
}

\author{
Kun-Jun Hwang, Jin-Woo Park, Il Kim, and Chang-Sik Ha* \\ Department of Polymer Science and Engineering, Pusan National University, Busan 609-735, Korea
}

Gue-Hyun Kim

Applied Engineering Division, Dongseo University, Busan 617-716, Korea

Received October 17, 2005; Revised January 4, 2006

\begin{abstract}
In the present work, low density polyethylene (LDPE)/aliphatic polyester (APES)/organoclay ternary nanocomposites were prepared. In particular, the effect of a compatibilizer, polyethylene-graft-maleic anhydride (PE- $g$-MAH), on the morphology and properties of the ternary nanocomposites was investigated. LDPE/APES/ organoclay nanocomposites were prepared through melt intercalation method using two different kinds of organoclay. The dispersibility of silicate clays in the nanocomposites was investigated by X-ray diffraction and atomic force microscopy. The ternary nanocomposites showed higher tensile properties than the LDPE/APES blend did. The dispersibility and properties of nanocomposites containing Cloisite $30 \mathrm{~B}$ were better than those of the nanocomposites containing Cloisite 20A. Unlike Cloisite 20A, hydroxyl groups in the intercalants in Cloisite 30B interlayer underwent a certain polar interaction with the carboxyl group of APES, favoring the intercalation of APES chains and the formation of LDPE/APES/Closite30B nanocomposites. However, the introduction of the polar hydroxyl groups also enhanced the interaction with the silicate surface at the same time, thereby rendering somewhat difficult the replacement of the surface contacts by LDPE chains, and impeding the extensive intercalation and further exfoliation of Cloisite 30B in the LDPE/APES matrix. The compatibilizer enhanced the intercalation of the polymer chain inside the clay gallery and thus improved the mechanical properties of the ternary nanocomposites. Rheological measurements of the nanocomposites via frequency sweep experiment indicated a certain interaction between the clay platelet and the polymer molecules in the melted state.
\end{abstract}

Keywords: biodegradable, nanocomposite, compatibilizer, low density polyethylene, aliphatic polyester.

\section{Introduction}

Low-density polyethylene (LDPE) is one of the most widely used polymers and extensively used in packaging materials. Most light weight plastic packaging materials are disposed after one-time application. Plastic wastes have adverse effects on the environment. Legislative threats and increasing public concern about garbage crisis have generated much interest in biodegradable packaging materials.

Two different approaches for the production of biodegradable packaging materials are currently being studied: One is the development of completely biodegradable packaging materials. The other is the development of partially biodegradable polymers obtained by blending non-biodegradable polymer and biodegradable polymers. In present technology, partially biodegradable polymers are more useful than com-

*Corresponding Author. E-mail: csha@pusan.ac.kr pletely biodegradable ones because of the economic advantages and better properties. ${ }^{1}$ For these reasons, there is an urgent need for the development for green polymeric materials that would not involve the use of toxic components in their manufactures, and could allow degradation via a natural composting process. In this sense, polymer-layered silicate (PLS) nanocomposites based on biodegradable polymers were found to be very attractive due to the environmentally friendly nature of clay. So far reported biodegradable polymers for the preparation of nanocomposites are polylactide, poly( $\varepsilon$-caprolactone), and aliphatic polyester (APES), etc. However, there are very few works on the PLS based on polymer blends containing a biodegradable polymer as one component.

Synthetic biodegradable APES, which are synthesized from diol and dicarboxylic acid through condensation polymerization, are known to be completely biodegradable in soil and water and their properties are closely resembled to 
LDPE. ${ }^{2}$ To add partial biodegradability to LDPE, LDPE and APES were blended in this study.

To improve mechanical properties of LDPE/APES blends, we adapted the PLS techniques. Melt intercalating polymers into the layered silicates of clay has been proven to be an excellent technique to prepare PLS nanocomposites. ${ }^{3-5}$ With only a few percent of clay, PLS exhibits greatly improved mechanical, thermal and barrier properties compared with the pristine polymers. ${ }^{6}$ Since silicate itself is environmentally friendly, these nanocomposites can preserve environmental gains and improve the mechanical strength and water sensitivity.

However, the incompatibility between hydrophilic APES and hydrophobic polyolefin often leads to the larger nondegradable residues and diminished mechanical properties. ${ }^{1}$ To enhance the compatibility between two immiscible polymers, a compatibilizer can be used. Also the compatibilizer used in the LDPE/APES/organoclay hybrids is expected to help LDPE to intercalate into the clays.

In the present study, LDPE/APES/organoclay ternary nanocomposites were prepared through melt intercalation method. Polyethylene-graft-maleic anhydride (PE- $g$-MAH) was used as the compatibilizer and its effect on mechanical properties and nanostructure of the LDPE/APES/organoclay hybrids were examined.

\section{Experimental}

Materials. Two kinds of organically modified montmorillonite (MMT), Cloisite 30B and Cloisite 20A, (Southern Clay Products) were used in our study. The intercalants are methyl, tallow bis-2-hydroxyethyl quaternary ammonium (MT2EtOH) for Cloisite 30B and dimethyl, dihydrogenated tallow quaternary ammonium (2M2HT) for Cloisite $20 \mathrm{~A}$. The chemical structures of the intercalants are depicted in Figure 1, where $\mathrm{T}$ is predominantly an octadecyl chain with smaller amounts of lower homologues (approximate composition: $\sim 65 \% \mathrm{C} 18, \sim 30 \% \mathrm{C} 16, \sim 5 \% \mathrm{C} 14)$ and $\mathrm{HT}$ is hydrogenated tallow. SK Chemicals' SKYGREEN ${ }^{\circledR}-2109$, poly(butylene succinate-co-adipate), (MI(ASTM D 1238)= $14 \mathrm{~g} / 10 \mathrm{~min}$ ), is a completely biodegradable aliphatic polyester (APES) resin. Low density polyethylene (LDPE, MI $($ ASTM D 1238) $=3.0 \mathrm{~g} / 10 \mathrm{~min})$, which is appropriate for packaging usage, was supplied by Hyundai Petrochemical Co., Korea. Polyethylene-graft-maleic anhydride (PE-gMAH, Aldrich) was used as a compatibilizer.

Preparation of LDPE/APES/Organoclay Nanocomposites. Organoclay (Cloisite 30B or Cloisite 20A), LDPE, APES, and PE- $g$-MAH were dried under vacuum at $80^{\circ} \mathrm{C}$ for at least $24 \mathrm{hrs}$. Dried LDPE, APES, Cloisite 30B (or Cloisite 20A) were mixed in a Haake Rheocord mixer at $140{ }^{\circ} \mathrm{C}$ with $100 \mathrm{rpm}$ of a rotor speed for $20 \mathrm{~min}$. The feed ratios of LDPE/APES/organoclay were 65/30/5, and LDPE/ APES/organoclay/PE- $g$-MAH were $60 / 30 / 5 / 5$ by weight.
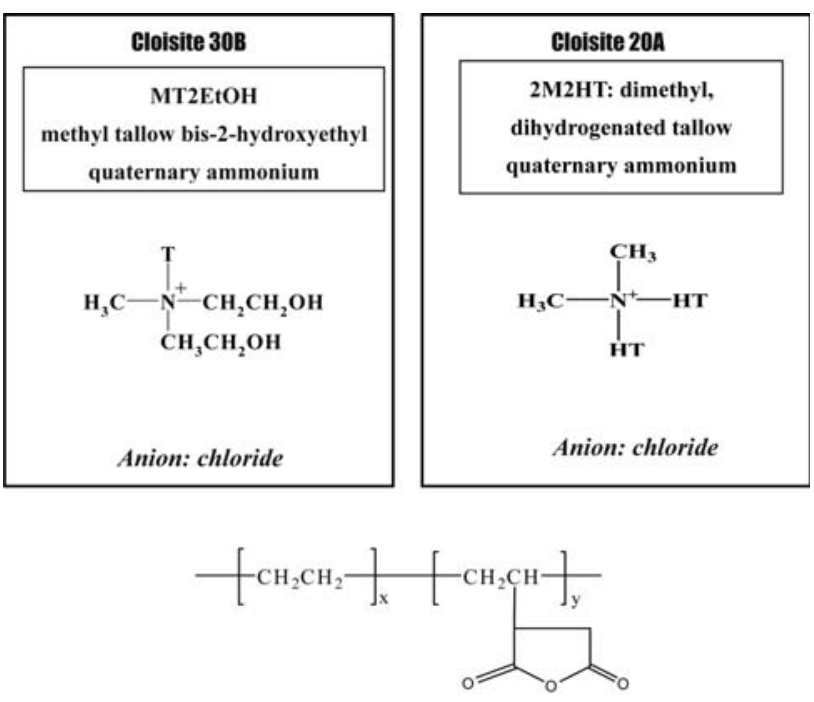

PE-g-MAH

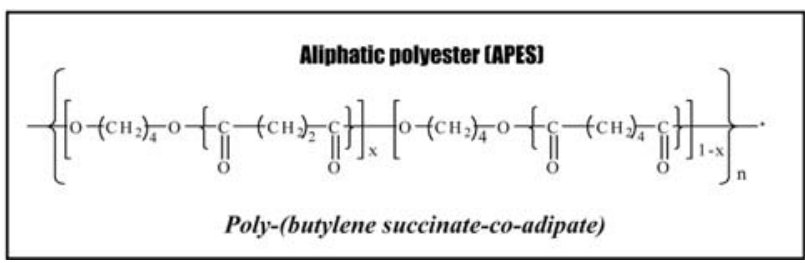

Figure 1. Chemical structures of materials used in this work.

The organoclay and compatibilizer content was fixed at $5 \mathrm{wt} \%$. For comparison, the LDPE/APES blend of 60/40 composition by weight was prepared in a similar way. The composition of various samples is summarized in Table I. Samples were then injection molded using a CS-183 MMX Mini-MAX molder (Custom Scientific Instruments, Inc.) to get dog-bone shaped specimens for characterization and property measurements. The melting and molding temperature was 155 and $160^{\circ} \mathrm{C}$, respectively. The specimens were placed in tightly sealed PE bags to prevent moisture absorption before measurements.

Measurements. XRD patterns were obtained using a Rigaku D/max 2200H X-ray diffractometer (40 kV, $50 \mathrm{~mA})$. The scanning rate is $0.5 \% \mathrm{~min}$. The basal spacing of the sili-

Table I. Composition of Various Samples

\begin{tabular}{lcccc}
\hline $\begin{array}{c}\text { Sample } \\
\text { Code }\end{array}$ & $\begin{array}{c}\text { LDPE } \\
(\mathrm{wt} \%)\end{array}$ & $\begin{array}{c}\text { APES } \\
(\mathrm{wt} \%)\end{array}$ & $\begin{array}{c}\text { PE- } g \text {-MAH } \\
\text { (wt\%) }\end{array}$ & $\begin{array}{c}\text { Organoclay } \\
(\mathrm{wt} \%)\end{array}$ \\
\hline LA & 60 & 40 & - & - \\
LA20A* $^{*}$ & 65 & 30 & - & 5 \\
LA30B* $^{*}$ & 65 & 30 & - & 5 \\
LA20AMAH* $^{*}$ & 60 & 30 & 5 & 5 \\
LA30BMAH* & 60 & 30 & 5 & 5
\end{tabular}

*20A and 30B means that Cloisite 20A and Cloisite 30B was used as organoclays, respectively. 
cate layer, $d$, was calculated using the Bragg's equation, $n \lambda$ $=2 d \sin \theta$. For AFM (Digital instruments, Nanoscope III) measurements, the cantilever used in the present study was a $\mathrm{V}$-shaped one mounted a quadrangular pyramid silicon nitride $\left(\mathrm{Si}_{3} \mathrm{~N}_{4}\right)$ micro-tip at the end. A bending spring constant of the cantilever was $0.022 \mathrm{~N} / \mathrm{m}$. In the AFM observations, a feedback loop kept the vertical position of the tip constant by moving the sample surface up and down with the piezoelectric scanner (phase mode). The images were taken with $1 \times 1 \mu \mathrm{m}^{2}$ scanners. The AFM observation was carried out in the air. For SEM measurements with a HITACHI instrument (model S-4200), the fractured samples were prepared in liquid nitrogen. The surfaces of the specimens were coated with gold to avoid charging under the electron beam.

\section{Results and Discussion}

Microstructure. Figures 2 and 3 show the XRD patterns of the LDPE/APES/organoclay nanocomposites. The XRD patterns of Cloisite 20A and Cloisite 30B revealed the diffraction peaks at $2 \theta=3.51^{\circ}$ and $2 \theta=5.28^{\circ}$ respectively, meaning that the layer distance of Cloisite 20A and Cloisite 30B were 2.5 and $1.85 \mathrm{~nm}$, respectively. The $d_{001}$ peak of the clay has been shifted to low angle, corresponding to an increase in $d$-spacing from 2.5 to 3.2 and $3.34 \mathrm{~nm}$ for LA20A (LDPE 65/APES 30/Closite20A 5) and LA20AMAH (LDPE 60/APES 30/Closite20A 5/PE-g-MAH 5) samples, respectively. And the $d_{001}$ peak of the clay has been shifted to low angle from 1.85 to 2.98 and $3.13 \mathrm{~nm}$ for LA30B (LDPE 65/ APES 30/Closite30B 5) and LA30BMAH (LDPE 60/APES 30/Closite30B 5/PE-g-MAH 5) samples, respectively. The higher basal spacing of clay in the nanocomposite as compared to virgin organoclay is due to the intercalation of polymer chains inside the clay layers.

In order for a polymer to be fully intercalated into the organoclay tactoids, it is imperative that the surface polarities of the polymer and organoclay be matched. ${ }^{4}$ Meanwhile, polar-type interactions are also critical for the formation of intercalated and especially exfoliated nanocomposites via polymer melt intercalation. ${ }^{7}$ Unlike the lack of strong polar interactions between the ammonium cation of Cloisite 20A and matrix polymer (LDPE /APES), hydroxyl groups in the intercalants in Closite 30B interlayer have a strong polar interaction with carboxyl group of APES, favoring the intercalation of APES chains and the formation of LDPE/APES/ Closite30B nanocomposites. ${ }^{8}$ Therefore, the degree of intercalation for the LDPE/APES/Cloisite 30B hybrids is higher than that for the LDPE/APES/Cloisite 20A. However, the introduction of the polar hydroxyl groups also enhances the interaction with the silicate surface at the same time. So replacement of the surface contacts by LDPE chains will be a little difficult, impeding the extensive intercalation and further exfoliation of Cloisite 30B in the LDPE/APES matrix.

It is noteworthy that the degree of intercalation in the

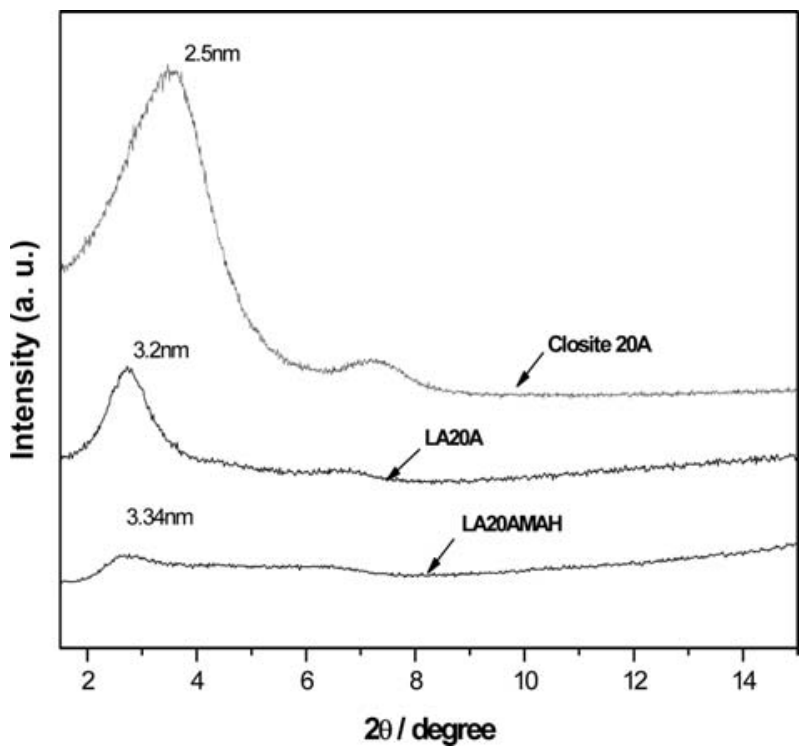

Figure 2. XRD patterns of LDPE/APES/Cloisite 20A nanocomposites with and without compatibilizer.

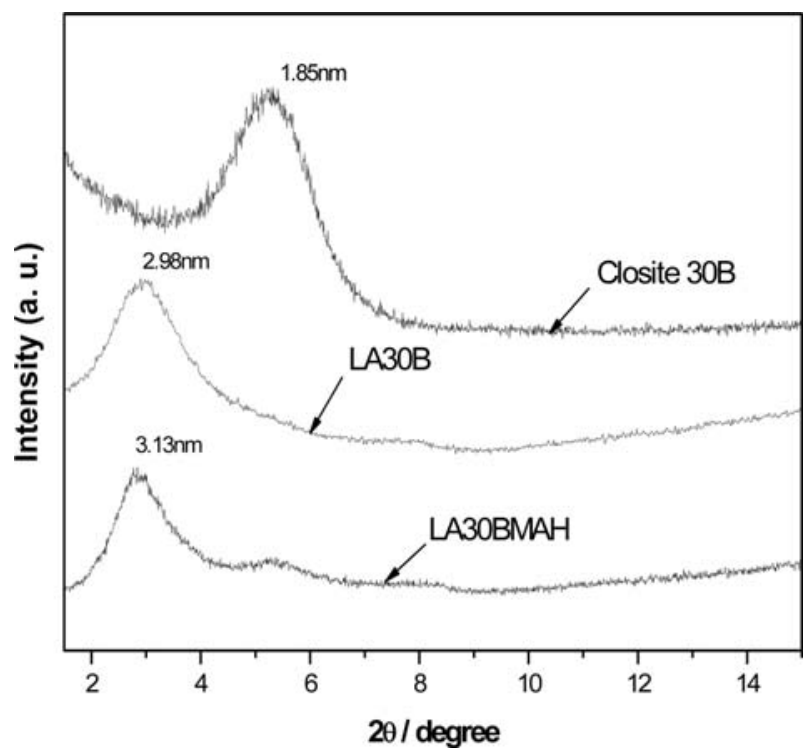

Figure 3. XRD patterns of LDPE/APES/Cloisite 30B nanocomposites with and without compatibilizer.

LDPE/APES/organoclay/PE- $g$-MAH nanocomposites is higher compared to the LDPE/APES/organoclay nanocomposites for both Cloisite 20A and Cloisite 30B, due to the compatibilizing effect of PE- $g$-MAH.

AFM is used to directly view the structure of nanocomposites, with the emphasis on the dispersion of the layered silicate fillers in the LDPE/APES matrix. Here, the tapping mode AFM is applied to get sufficient mechanical (intrinsic) contrast between the two components (hard inorganic silicate filler and soft polymer matrix). In the case of poly- 
mer nanocomposites, in Figures 4 and 5, it can be seen that the silicate particles (visible as dark lines in phase image) in the nanocomposites are finely dispersed in the LDPE/APES matrix with an average length from 0.1 to $1 \mathrm{~mm}$ for both organoclays. No significant exfoliated clay platelets are detected as was consistent with the XRD measurements. For the LDPE /APES blend, such silicate layers (dark lines) were not detected. As shown in Figure 5, the average distance between the stacks of layers evaluated from overall picture is around $3 \mathrm{~nm}$ (3.906 $\mathrm{nm}$ for LA20A and $2.930 \mathrm{~nm}$ for LA30B,

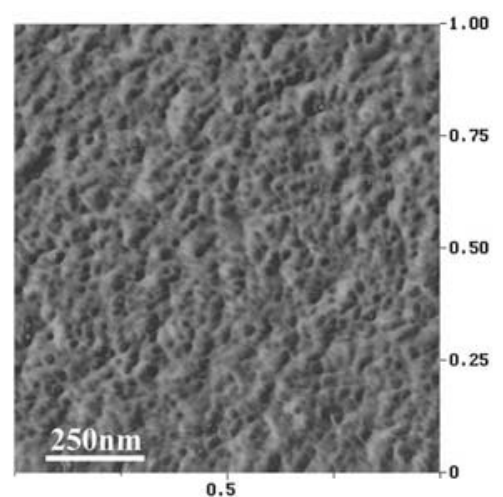

(a) LA

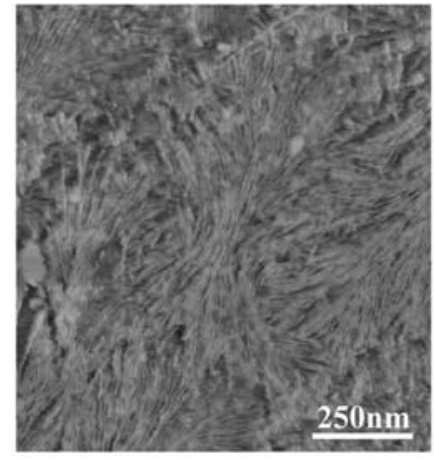

(b) LA30B

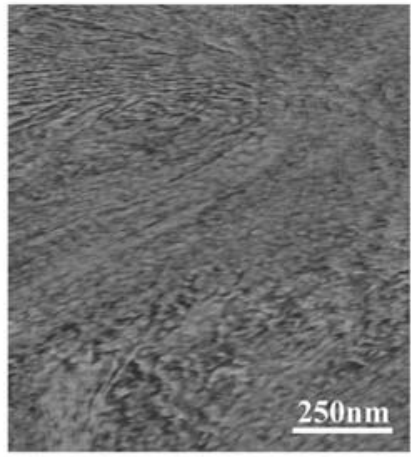

(c) LA20A

Figure 4. Tapping-mode AFM images of LDPE/APES/organoclay nanocomposites (phase images $1 \times 1 \mu \mathrm{m}^{2}$ ).
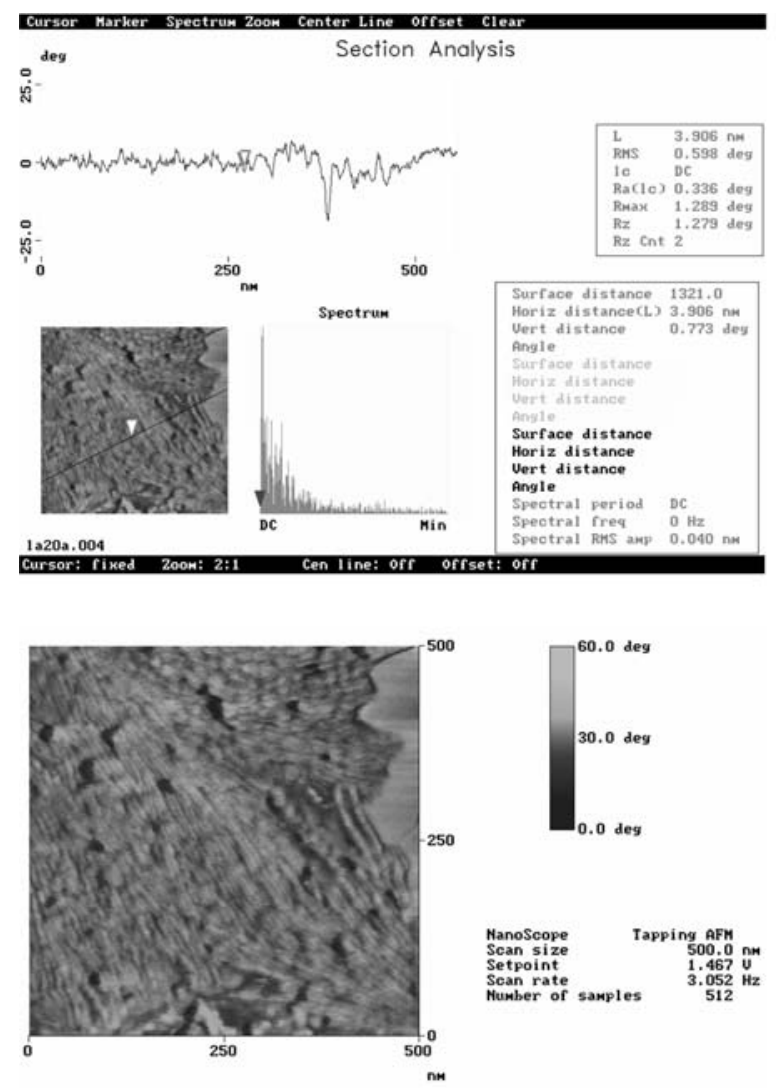

(a)
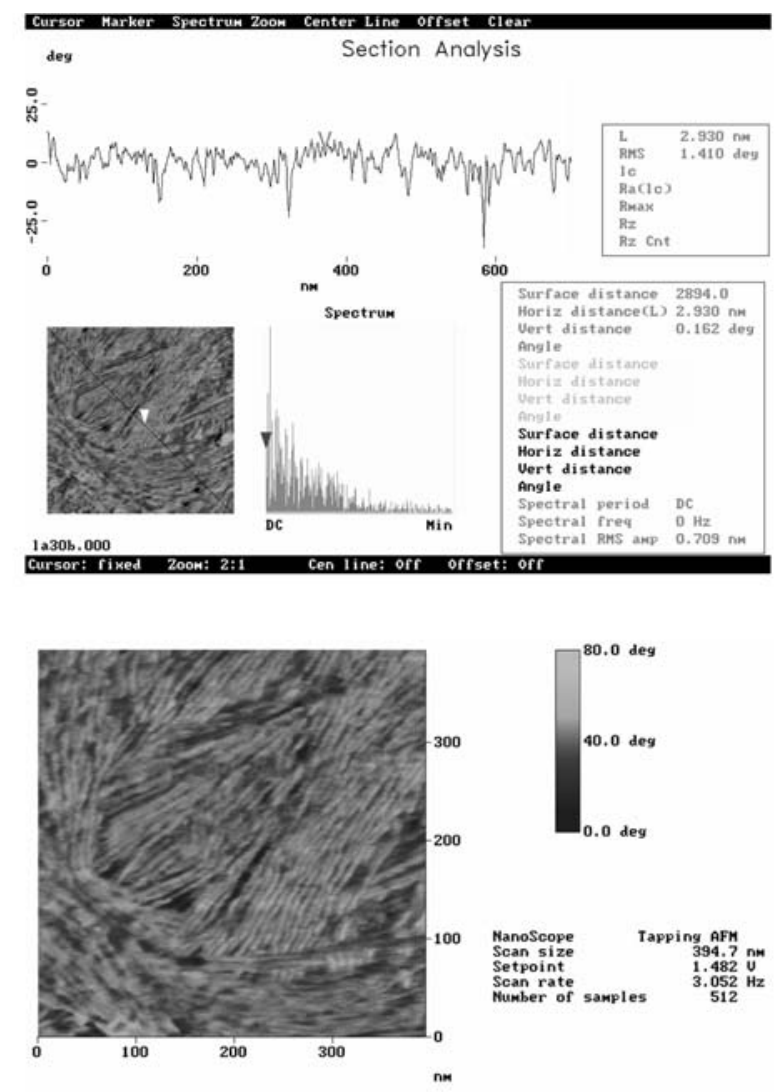

(b)

Figure 5. Section analysis results from the AFM images of (a) LDPE/APES/Cloisite 20A and (b) LDPE/APES/Cloisite 30B nanocomposites. 
respectively) from the section analysis of the AFM images. This is in good agreement with XRD results where basal (001) reflection is observed. The surface roughness ranged from 3.4 to $4.6 \mathrm{~nm}$ for the LDPE/APES blend and the LDPE/ APES/Cloisite 20A and LDPE/APES/Cloisite 30B nanocomposites.

No noticeable differences were observed for the AFM images of the LDPE/APES/organoclay/PE- $g$-MAH nanocomposites in comparison to those of the ternary nanocomposites without the compatibilizer regardless of the organoclay types.

Mechanical Properties. Figure 6 shows that the mechanical properties of the prepared nanocomposites. The tensile strength of LDPE and APES are 14.2 and 13.2 MPa, whereas the tensile modulus of LDPE and APES are 12.3 and 10.2 MPa. The tensile strength and modulus of LDPE/APES blend of 60/40 composition by weight are 7.3 and $28.4 \mathrm{MPa}$, respectively, while the tensile strength and modulus of LDPE/APES blend of 70/30 composition by weight are 9.2 and 29.3 MPa, respectively. The tensile properties of LDPE/ APES blends are deteriative in comparison to those of each component polymers. The LDPE/APES/organoclay nanocomposite, however, showed higher tensile strength and modulus than the corresponding LDPE/APES blends for both types of organoclay. The intercalation of the polymer chains inside the silicate layers leads to an increase in the surface area of interaction between the clay and polymer matrix, which results in the improvement of modulus in the entire prepared nanocomposites. Unexpectedly, however, there was not much difference in the tensile properties in between LDPE/APES/Cloisite 30B nanocomposite and LDPE/APES/ Cloisite 20A nanocomposite, though Cloisite 30B containing hydroxyl group has more polar interaction with aliphatic polyester than Cloisite 20A. ${ }^{8}$ On the other hand, the LDPE/ APES/organoclay nanocomposites with a compatibilizer (PE-

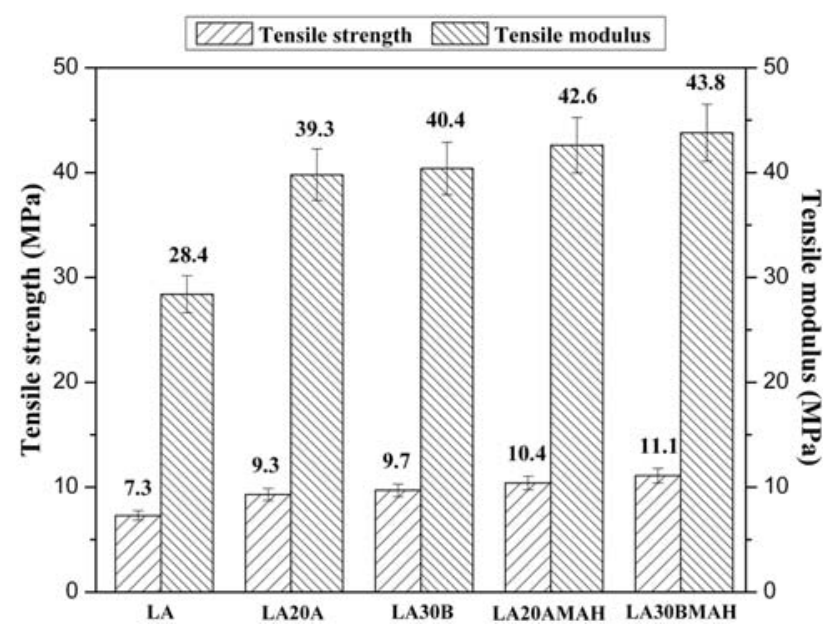

Figure 6. Mechanical properties of LDPE/APES/organoclay nanocomposites with and without compatibilizer.
g-MAH) show higher tensile strength and tensile modulus than LDPE/APES/organoclay nanocomposites without a compatibilizer, regardless of the organoclay types.

Figure 7 shows the SEM photographs of LDPE/APES/ organoclay nanocomposites containing Cloisite 20A or Cloisite 30B as well as the LDPE/APES blend, indicating the immiscibility of APES and LDPE in the blends and in the nanocomposites. For the nanocomposites, however, the bright particle-like morphology was observed, suggesting the polymer chain pulling out and broken point after elongation due to the presence of clays. Comparison of Figure 7(b) and 7(c) indicates that the LDPE/APES/Cloisite 30B nanocomposite shows finer particle-like morphology as the bright images than the LDPE/APES/Cloisite 20A nanocomposite does, due to the better compatibility and stronger interfacial interaction of APES with Cloisite 30B than with Cloisite 20A as reported in our previous work. ${ }^{8}$ The better mechanical properties (such as tensile strength and tensile modulus) for the nanocomposites containing Cloisite 30B than the nanocomposites containing Cloisite 20A, though the difference is not so large, may be related in part to such morphological differences.

Melt Rheological Behavior. In polymer processing, rheological properties are very important in order to properly control the process. In general, rheological properties

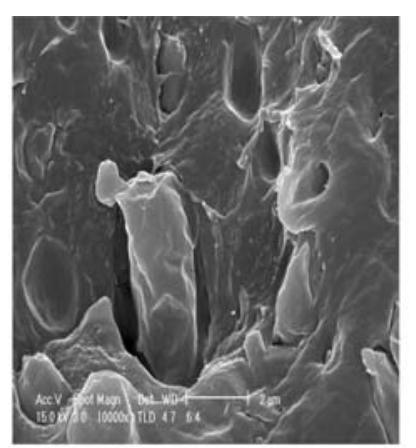

(a) LA

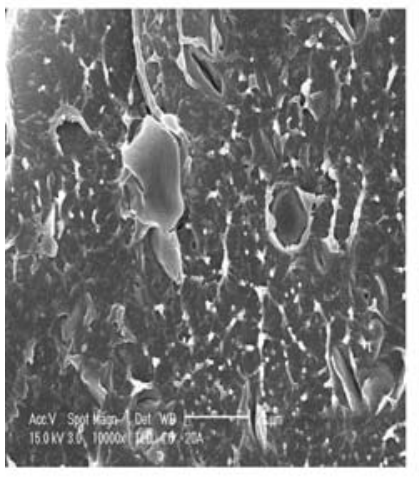

(b) LA20A

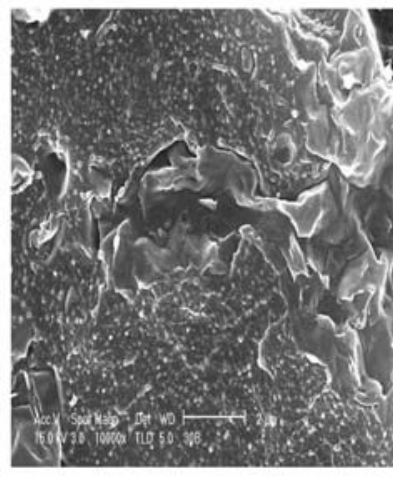

(c) LA30B
Figure 7. SEM photographs of LDPE/APES/organoclay nanocomposites fractured in liquid nitrogen. 
are largely influenced by the morphology of blends. Hence, they are one of the comparable standards for determining compatibility between nonpolar LDPE and polar APES.

The storage modulus $\left(G^{\prime}\right)$ and loss modulus $\left(G^{\prime \prime}\right)$ of LDPE/ APES blend and LDPE/APES/organoclay nanocomposites with and without a compatibilizer are shown in Figures 8 to 11. Over the entire studied frequency range the storage and loss modulus of the nanocomposites are substantially higher but the terminal slopes of the storage modulus are lower than that of their pristine blend counterpart. The storage modulus and loss modulus increase with the increased frequency of all the studied samples. This is due to the fact that at low frequency, time is large enough to unraveling of the entanglements so a large amount of relaxation occur, resulting in

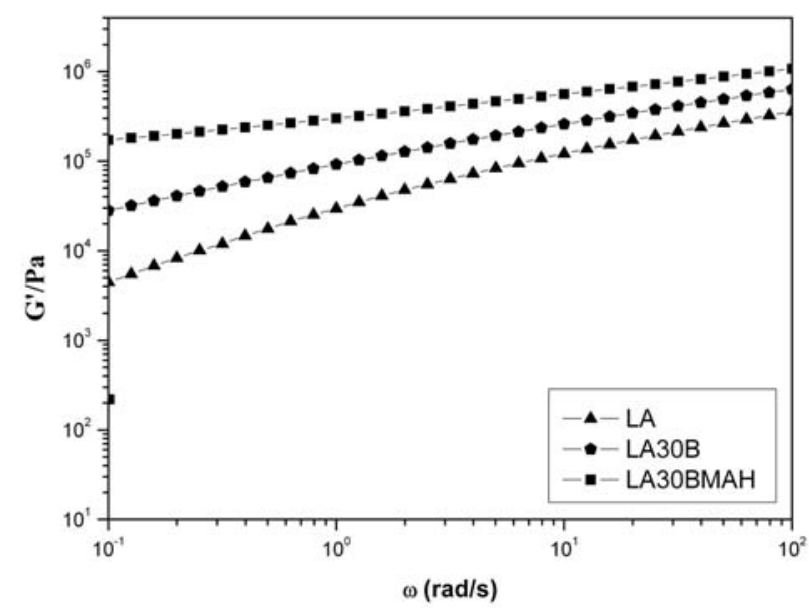

Figure 8. $G^{\prime}$ (storage modulus) vs frequency $(\omega)$ of LDPE/APES blend and LDPE/APES/Cloisite 30B nanocomposites with and without compatibilizer.

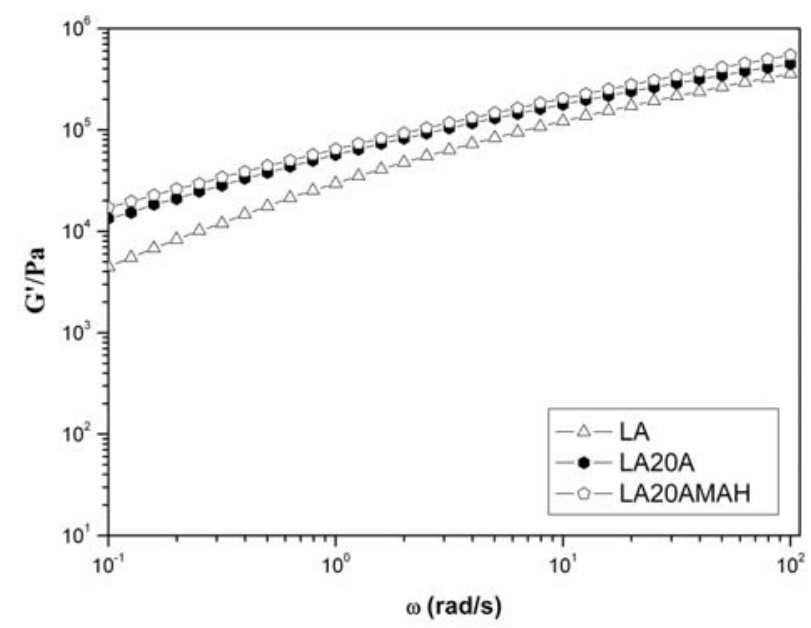

Figure 9. $G^{\prime}$ (storage modulus) vs frequency $(\omega)$ of LDPE/APES blend and LDPE/APES/Cloisite 20A nanocomposites with and without compatibilizer. a low value of storage and loss modulus. ${ }^{9}$ However when a polymer sample is deformed at large frequency the entanglement chains do not have time to relax, so modulus goes up. ${ }^{10}$ Figures 8 and 11 indicate that the storage modulus of the nanocomposites containing Closite 30B with the compatibilizer is higher than that without the compatibilizer whereas not much difference is observed in the storage modulus for the nanocomposite containing Cloisite 20A depending on the presence or absence of the compatibilizer. At $1 \mathrm{rad} / \mathrm{sec}$ the storage modulus of LA30BMAH is $58 \%$ higher than that of LA20AMAH whereas for LA30B it is $28 \%$ higher than that of LA20A. It indicates again that Closite 30B possesses stronger interactions with the polymer matrix. The higher $G^{\prime}$ and the smaller terminal slope over their pristine

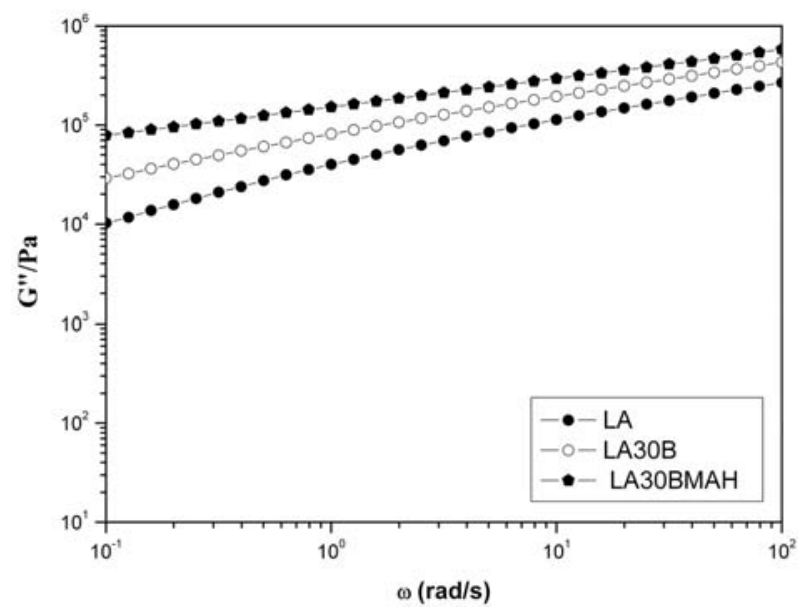

Figure 10. $G^{\prime \prime}$ (loss modulus) vs frequency $(\omega)$ of LDPE/APES blend and LDPE/APES/Cloisite 30B nanocomposites with and without compatibilizer.

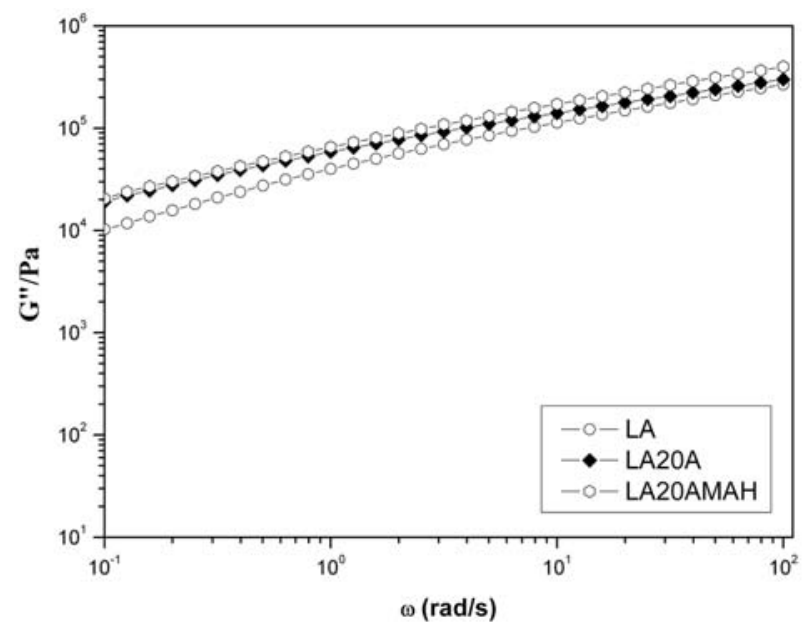

Figure 11. $G^{\prime \prime}$ (loss modulus) vs frequency $(\omega)$ of LDPE/APES blend and LDPE/APES/Cloisite 20A nanocomposites with and without compatibilizer. 
equivalent are usually observed in nanocomposite systems. ${ }^{11-13}$

The changes in the complex viscosity $\left(\eta^{*}\right)$ of nanocomposites and their pristine blend counterpart is shown in Figures 12 and 13. It is observed in Figures 12 and 13 that the complex viscosity decreases with increased frequency. This is due to the shear thinning behaviors of the polymer nanocomposites and their pristine blend equivalent at the melted state. ${ }^{10}$ The viscosity of the nanocomposites is higher than their pristine blend counterpart for both types of organoclay. At $1 \mathrm{rad} / \mathrm{sec}$ the viscosity of the LA30BMAH is $58 \%$ higher than that of LA20AMAH, whereas it is $29 \%$ higher in case of LA30B (as compared to LA20A). The higher melt viscosity of the nanocomposites containing Cloisite 30B than Cloisite 20A is attributed to the interaction of Closite 30B

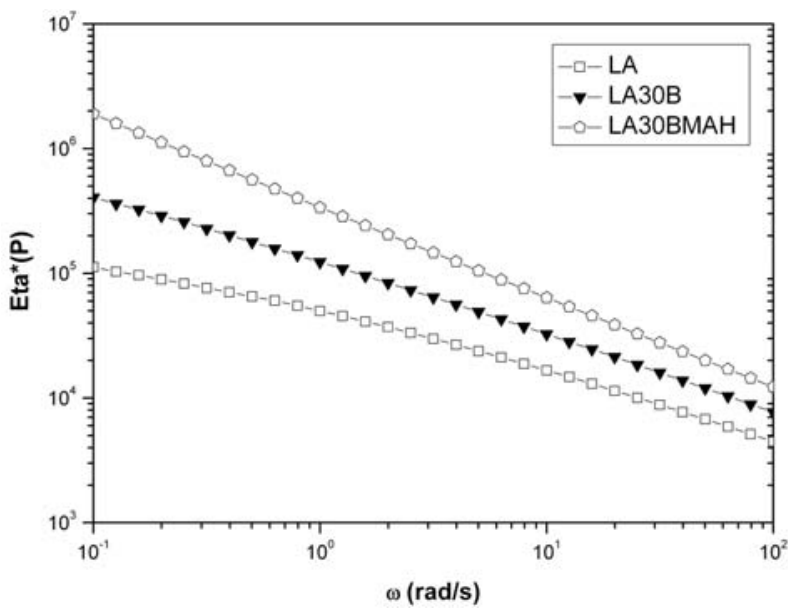

Figure 12. $\eta^{*}$ (complex viscosity) vs frequency $(\omega)$ of LDPE/ APES blend and LDPE/APES/Cloisite 30B nanocomposites with and without compatibilizer.

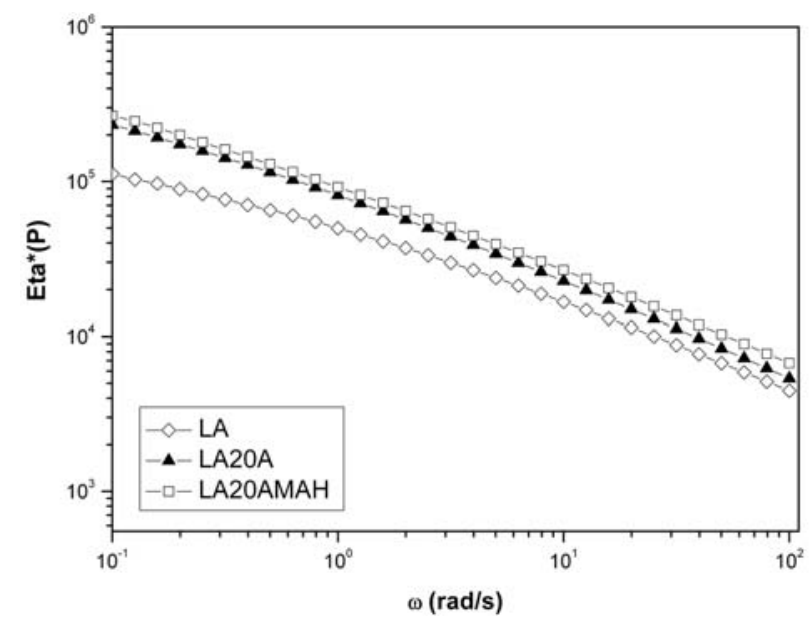

Figure 13. $\eta^{*}$ (complex viscosity) vs frequency $(\omega)$ of LDPE/ APES blend and LDPE/APES/Cloisite 20A nanocomposites with and without compatibilizer. and polymer matrix.

Before concluding, it should be mentioned that there exist many complex problems in an alloy nanocomposites, simultaneously or independently, including the clay dispersion in matrix, domain coalescence and interfacial tension change by adding organoclays, and the migration of the clay particle from a phase to another phase due to the interfacial tension, etc. Furthermore, more systematic experimental approach is needed to monitor compatibility change except to presenting intercalation behavior through multi-technique experiments including more systematic XRD and TEM experiments. The properties of polymer/clay nanocomposites would be highly governed by the change of intercalation behavior. However, the properties of the mixture system of the present work cannot be simply understood only in terms of intercalation behavior, since an increase of clay gallery by polymer intercalation may be found even for incompatible situation such as melt intercalation of clay with polystyrene. ${ }^{7}$ Therefore, further research on more detailed and theoretical interpretation on the compatibilizing effect of PE- $g$-MAH for the LDPE/APES/organoclay nanocomposites is now underway and will be reported elsewhere.

\section{Conclusions}

LDPE/APES/organoclay ternary nanocomposites were prepared using PE- $g$-MAH as a compatibilizer. The compatibilizer enhances the mechanical properties of the LDPE/ APES/organoclay hybrids. The dispersibility of organoclays in the hybrid was investigated by using an X-ray diffraction and atomic force microscope. Also, homogeneous dispersion of the organoclay layers in LDPE/APES blend was verified by AFM and SEM analysis. Enhancement of storage modulus (in the melted state) and decrease in terminal slope of the nanocomposite (over its pristine equivalent in the frequency sweep experiment) indicate to the fact that an interaction exists between clay platelet and polymer molecules in the melted state. In summary, the compatibilizing effect of PE$g$-MAH was observed in the mechanical property and dispersion of organoclays for the LDPE/APES blend.

Acknowledgements. The work was supported by the Pusan National University Research Grant in 2005.

\section{References}

(1) I. M. Thakore, S. Desai, B. D. Sarawade, and S. Devi, Eur. Polym. J., 37, 151 (2001).

(2) T. Fujimaki, Polym. Degrad. Stab., 59, 209 (1998).

(3) H. M. Jeong and Y. T. Ah, Macromol. Res., 13, 102 (2005).

(4) S. Y. Park and Y. H. Cho, Macromol. Res., 13, 156 (2005).

(5) M. Xu, Y. S. Choi, K. H. Wang, J. H. Kim, and I. J. Chung, Macromol. Res., 11, 410 (2003).

(6) A. Okada and A. Usuki, Mater. Sci. Eng., C3, 109 (1995). 
(7) R. A. Vaia and E. P. Giannelis, Macromolecules, 30, 8000 (1997).

(8) S. R. Lee, H. M. Park, H. T. Lim, T. K. Kang, X. Li, W. J. Cho, and C. S. Ha, Polymer, 43, 2495 (2002).

(9) C. D. Muzny, B. D. Butler, H. J. Hanley, F. Tsvetkov, and D. G. Peiffer, Mater. Lett., 28, 379 (1996).

(10) V. Pasanovic-Zujo, R. K. Gupta, and S. N. Bhattacharya,
Rheol. Acta., 43, 99 (2004).

(11) Y. H. Hyun, S. T. Lim, H. J. Choi, and M. S. Jeon, Macromolecules, 34, 8084 (2001).

(12) J. K. Mishra, K. J. Hwang, and C. S. Ha, Polymer, 46, 1995 (2005).

(13) R. S. Sinha, K. Okamoto, and M. Okamoto, Macromolecules, 36, 2355 (2003). 